\title{
Secure Data Access Computing Model on Mobile Cloud Data using Fusion of Finger Print and Face biometric authentication based on Discrete Combinational models
}

\author{
A.Amali Mary Bastina ${ }^{1}$ and N.Rama ${ }^{2}$ \\ ${ }^{1}$ Department of computer Science, Loyolo College, Chennai, India \\ ${ }^{2}$ Department of computer Science Presidency College, Chennai, India
}

\section{ABSTRACT}

Secure data access model on Mobile cloud data has received a lot of attention recently and is viewed as a promising trait. Biometric based authentication to secure access of the mobile cloud data have undergone fast development towards data protection and preventing malicious data threats. In this work, multimodal biometric based authentication technique as fusion model has been projected for data security. The key characteristics of finger print and face patterns include its uniqueness to each individual, unforgettable, nonintrusive and cannot be taken by an unauthorized person. In related methods, feature selection methods explore intrinsic finger print and face on single feature method, but their performance remains undesirable in terms of computational cost. However the extracted features from the both finger print and face pattern are huge with high redundancy. On employment of fusion concept on feature extraction techniques through weighted average strategies, equal error rate is minimized to obtain the optimum weight. In this paper, we propose a combinational model composed differential evolution technique to enhance the recognition of finger print and face patterns to authenticate the user towards data access. The system has been trained in selecting high relevant features on using the extraction techniques such as principle component analysis and linear discriminant analysis. Feature fusion carried out as concatenation on PCA and LDA technique using discriminant correlation analysis feeds the proposed feature selection models by optimal subset of features. The proposed system uses differential model to determine the less no of optimal features for multimodal authentication. Multimodal authentication performed using Multivariate linear regression on less no of optimal features. The analysis of empirical results shows that proposed system produces the best accuracy reflected by 100 percent accuracy on comparing with existing single model biometric authentication models. Further Proposed model has been evaluated and the results shows remarkable efficiency with existing state of art approaches.

KEY WORDS: FINGER PRINT RECOGNITION, FACEDETECTION, PRINCIPLE COMPONENTANALYSIS, LINEAR DISCRIMINANT ANALYSIS, MULTIVARIATE LINEAR REGRESSION, DIFFERENTIAL EVOLUTION, DISCRIMINANT CORRELATION ANALYSIS.

\section{INTRODUCTION}

Currently, the rapidly advanced cloud-assisted mobile multimedia services motivate the owner to share their multimedia data via the cloud platform. However, the data

Biosc Biotech Res Comm P-ISSN: 0974-6455 E-ISSN: 2321-4007

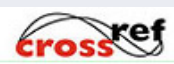

Identifiers and Pagination

Year: 2021 Vol: 14 No (7) Special Issue

Pages: 292-298

This is an open access article under Creative

Commons License Attribn 4.0 Intl (CC-BY). DOI: http://dx.doi.org/10.21786/bbrc/14.7.64 security and privacy concerns would arise while enjoying the rich computation and storage resources for saving the overhead of mobile devices. In particularly, once the multimedia data is outsourced to the cloud, the data owner has to rely on the cloud server to enforce the access control. But the cloud server cannot be fully trusted and it would acquire the owner's privacy and recommend advertisement endlessly. A well adoptable approach is to encrypt the multimedia data before offloading it to the cloud. Whereas, the adoption of traditional cryptology or identity-based encryption might lead to complicated key distribution and management here are various biometric technologies currently available, such as iris, fingerprint, face, voice, and finger-vein.

\section{Article Information}

Received: $11^{\text {th }}$ May 2021 ccepted after revision: $19^{\text {th }}$ July 2021 
Fusion of Fingerprint (F. Liu et. al. (2014)) and Face (J. Yang et. al. (2017)) are easy to be acquired and difficult to be forged because of existence inside. However, the clear observation of fingerprint and face patterns is affected by shades and changes in illumination, which causes the degradation of recognition accuracy. The existing featurebased methods for fingerprint and face recognition, such as local binary pattern (B. A. Rosdi et. al. (2011)) and Discriminative Binary Descriptor (Fan ZXu Y et. al. (2011)) have reduced recognition accuracy if noise caused by shades and changes in illumination in images are not properly corrected during pre-processing. Feature extraction method have been employed to explore intrinsic finger features but their remains undesirable on extraction of large features which leads to high redundancy and high computational cost. Taking aforementioned consideration into account, a new combinational model has been proposed in this paper.

In order to enhance the recognition rate of fingerprint and face based authentication system, fusion concept has been employed on feature fusion and fusion selection separately using various techniques. Initially four extraction techniques such as principle component analysis (Fan Z.Xu et. al. (2011)) and linear discriminant analysis (Kumar et. al. (2008)) have been used to select high relevant features. Selected features undergo concatenation on PCA and LDA technique for feature fusion. Discriminant Correlation Analysis has been provided with Features of the biometric and fusion of multimodal biometric has been attained. Further feature selection models have been proposed to generate optimal subset of features using differential evolution. Finally multivariate linear regression has been used to prediction of the user towards data access on less no of optimal features. The rest of this paper is organized as follows. Section 2 presents the related work based Local Binary Patterns and Discriminative Binary Descriptor. The proposed model and its components are discussed in section 3. The experimental results and analysis are discussed in section 4. Finally, the conclusion of this work and future improvement are presented in section 5 .

2. Related works: In this section, finger vein recognition which has been carried through many feature based techniques such as Local Binary pattern and Discrete Binary Patterns has been discussed in detail on various aspects, detailed process description is as follows

\subsection{Finger Print Recognition using Local Binary} Patterns: In this model, finger print features are extracted with line patterns orientation on horizontally and vertically with most discriminative ability on texture representation. It is then analysed with the discriminative ability of LBP histograms from different orientations(Shazeeda et. al.(2016)). Finally matching score-level fusion using sum rule is applied to achieve the final matching score for the recognition to have better performance.

\subsection{Face Recognition using Discriminative Binary} Descriptor: In this model, feature are extracted using LBP or Gabor descriptors, extracted feature has been mapped using discriminative feature learning method to enhance the discriminative ability of the local features extracted(Haiying et. al.(2017)). The discrimination of the feature is attained through multi-directional pixel difference vectors for each pixel and its neighbouring pixel. Finally finger vein recognition is carried using classifier on the histogram of the finger vein features.

3. Proposed model: The Proposed model for fusion of finger print and face authentication for secure access of mobile cloud data consist of following phases, such as feature extraction, feature reduction, feature fusion, Feature selection and finally feature classification on the optimal set of features. As initial step, finger print and face image acquired will undergo pre-processing includes the steps of binary thresholding, in-plane rotation compensation, and detection of finger and face region-of-interest. The overall flowchart of the multimodal authentication system for secure data access has proposed in this study is shown in Figure 1.

Figure 1: Architecture of the Proposed Combinational model for Multimodal Authentication

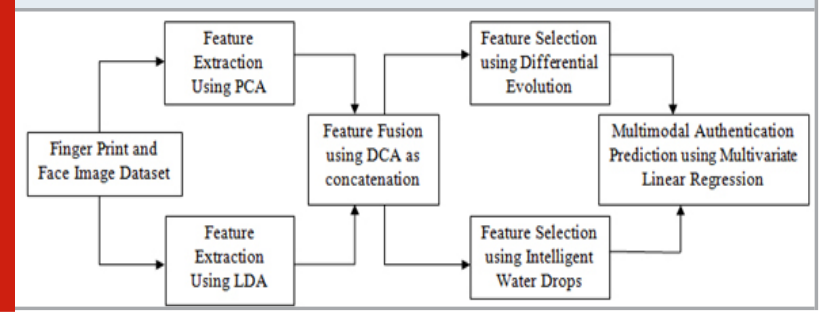

Pre-processed image will undergo feature extraction with four techniques for selecting relevant features. Those extracted feature will be concatenated for feature fusion. Concatenated feature employed to feature selection to select optimal subset features for multimodal authentication. Differential Evolution is to determine the less no of optimal features for fusion of biometric recognition. Finally Multivariate Linear Regression classifier is used for feature classification or recognition.

3.1. Feature Extraction: The feature extraction phase aims to extract the relevant features of fingerprint and face through following dimensionality reduction and normalization technique such as.

3.1.1. Principle Component Analysis: Principal component analysis (PCA) is employed for feature extraction produces the most distinguishing features. PCA is implemented to generate a set of training data for the face and fingerprint image for recognition application that can be separated as far as possible and the same classes of patterns are compacted as close as possible. It is used for analyzing the image objects, identifying patterns and expressing the object to highlight their differences on face and fingerprint. It describes the object of the image in terms of variance. Each principle component of the image describes the greatest amount of variance. Since the patterns can be hard to find in data of high dimension, PCA can find these patterns in the data by reducing the number of dimensions, without much loss of information. Finally it consists of feature vectors of the fingerprint and face patterns. Given $M$ fingerprint or face 
images of size $\mathrm{N} x \mathrm{~N}$, first represent each image to a 1D vector $U$. Vector composed of variance values. Variance for particular object $\mathrm{X}$ in a image is computed as follows

$$
\operatorname{var}(\mathrm{x})=\frac{\sum_{i=1}^{n} \mathrm{a}(\mathrm{xi}-\mathrm{x})(\mathrm{xi}-\mathrm{x})}{n-1}
$$

Covariance is computed for the $\mathrm{X}$ and $\mathrm{Y}$ object of the face and fingerprint which changes together with mean for secure data access is as follows

$$
\operatorname{Cov}(\mathrm{x}, \mathrm{y})=\frac{\sum_{i=1}^{n} \mathrm{a}(\mathrm{xi}-\mathrm{x})(\mathrm{yi}-\mathrm{y})}{n-1}
$$

Covariance Matrix for secure data access is a $N^{*} \mathrm{~N}$ Matrix, where each element is given by

$$
\mathrm{M}_{\mathrm{ij}}=\operatorname{Cov}(\mathrm{x}, \mathrm{y})
$$

Eigen Vector of $\mathrm{M}_{\mathrm{ij}}$ is a vector composed of principle feature set with values as eigen value for classification or recognition.

3.1.2 Linear Discriminant analysis: Linear discriminant analysis is used to identify an optimal solution vector as the best projection direction based on the fisher criterion function so that after projection in this direction of the feature fusion, the homogeneous samples in the original data set composed of face and fingerprint image are as close as possible and the heterogeneous samples are separated as far as possible. LDA algorithm is essentially looking for the effective transformation feature vector. The optimal solution fusion vector is obtained on reduction of the feature space through usage of scatter matrix on face and fingerprint. Given $\mathrm{M}$ fingerprint and face images of size $\mathrm{N} x \mathrm{~N}$, first compute the mean vector for the image.

Mean Vector for Single face object Mi

$$
\begin{aligned}
& =\frac{1}{\mathrm{n}}\left(\begin{array}{l}
n \\
k
\end{array}\right) x^{k} \sum_{x \varepsilon C}^{n} \mathrm{x}, \mathrm{n}_{\mathrm{i}} \\
& \sum_{k=0}^{n}\left(\begin{array}{l}
n \\
k
\end{array}\right) x^{k} a^{n-k} \mathrm{~N}
\end{aligned}
$$

Scatter Matrix for with Vector Scatters of face

$$
\mathrm{F}_{\mathrm{w}}=\sum_{i=1}^{c} \mathrm{~s}_{\mathrm{i}} \frac{n i}{\mathrm{n}}
$$

Scatter Matrix for between vector Scatters

$$
\mathrm{F}_{\mathrm{b}}=\frac{1}{2}\left(\begin{array}{l}
n \\
k
\end{array}\right) x^{k} \sum_{i=1}^{c} \mathrm{P}_{\mathrm{i}} \mathrm{P}_{\mathrm{j}}\left(\mathrm{k}_{\mathrm{i}}-\mathrm{k}_{\mathrm{j}}\right)\left(\mathrm{k}_{\mathrm{i}}-\mathrm{k}_{\mathrm{j}}\right)^{\mathrm{T}}
$$

where $\left(\mathrm{k}_{\mathrm{i}}-\mathrm{k}_{\mathrm{j}}\right)$ is vector instance difference.

Covariance to the mean vector of fusion obtained is computed as

$$
\mathbf{C}_{\mathbf{i}}=\frac{1}{\mathrm{n}}\left(\begin{array}{l}
n \\
k
\end{array}\right) x^{k} \sum_{x \varepsilon C}^{1}\left(\mathrm{x}-\mathrm{k}_{\mathrm{i}}\right)\left(\mathrm{x}-\mathrm{k}_{\mathrm{i}}\right)^{\mathrm{T}}
$$

Total Covariance of Scatter matrixes to yield optimal fusion feature set is

$$
\mathbf{C}_{\mathbf{T}}=\frac{1}{\mathrm{~N}} \Sigma\left(\mathrm{k}_{\mathrm{i}}-\mathrm{k}_{\mathrm{j}}\right)\left(\mathrm{k}_{\mathrm{i}}-\mathrm{k}_{\mathrm{j}}\right)^{\mathrm{T}}
$$

Important consideration of LDA technique is to minimize $\mathrm{F}_{\mathrm{w}}$ while maximizing $\mathrm{F}_{\mathrm{b}}$ on the data for securing. This can be achieved by maximizing the ratio $\left|\mathrm{F}_{\mathrm{b}}\right| /\left|\mathrm{F}_{\mathrm{w}}\right|$. Further feature weight gets maximized when eigenvectors of $\mathrm{f}_{\mathrm{b}} \mathrm{f}_{\mathrm{w}}$ form the column vector of the projection matrix of fingerprint and face fused for secure user authentication.

$$
\mathrm{W}=\left[\mathrm{W}_{1}, \mathrm{~W}_{2} \ldots \ldots \ldots \mathrm{W}_{\mathrm{c}-1}\right]
$$

Where $\mathrm{W}_{\mathrm{i}}$ are the eigenvectors of the $\mathrm{f}_{\mathrm{b}}$ and $\mathrm{f}_{\mathrm{w}}$ corresponding to the set of decreasing eigenvalues $\lambda_{\mathrm{i}}$

Linear discriminant analysis has been employed to produce the optimal set of features through normalization on pair wise similarities of the correlated features extracted on the variance of the object and dimensionality reduction of face and fingerprint on estimating the linear combination of features on two or more vectors of multibiometric authentication.

3.2.Feature Level Fusion: The Primary focus of feature fusion for secure authentication is to combine relevant information from two or more feature vectors into a single one with more discriminative power than any of the input feature vectors of face and fingerprint biometric. Feature level fusion has been effective in combining perceptually salient image features extracted from the principle component analysis and linear discriminant analysis models in low dimensional space to yield concatenated feature using discriminant Correlation analysis (Mohammad Haghighat et. al. (2016)) on vector associations in the reduced feature set of face and fingerprint. These two homogenous vectors are processed pragmatically to produce the fused vector of same order.

$$
\mathrm{Y}_{\mathrm{PCA}}=\left[\mathrm{w}_{\mathrm{PCA} 1}, \mathrm{wP}_{\mathrm{CA} 2}, \mathrm{w}_{\mathrm{PCA} 3} \ldots \mathrm{w}_{\mathrm{PCAn}}\right]
$$

Where $\mathrm{Y}_{\mathrm{PCA}}$ is the vein feature Vector extracted by the PCA

$\mathrm{Y}_{\mathrm{LDA}}=\left[\mathrm{w}_{\mathrm{LDA} 1}, \mathrm{w}_{\mathrm{LDA} 2}, \mathrm{w}_{\mathrm{LDA} 3} \ldots . \mathrm{w}_{\mathrm{LDAn}}\right]$

Where $Y_{L D A}$ is the vein feature Vector extracted by the LDA

$\mathrm{n}$ is the number of training samples.

A new feature vector has been generated by serially combining or concatenating the Principle Component Analysis extracted features YPCA and corresponding linear discriminant Analysis extracted features YLDA on 
computation of the distance vector of APCA and dLDA of each vector extracted. These two vectors are combined according to combination rule by maximizing pair wise correlation. In this work vector combination rule employed is considered as sum of the feature vectors.

Combined Distance Vector by Mean Vector.

$$
\mathrm{d}=\left\{\frac{\mathrm{d} 1 \mathrm{PCA}+\mathrm{d} 1 \mathrm{LDA}}{2} \ldots \frac{\mathrm{dNPCA}+\mathrm{dNLDA}}{2}\right\}
$$

Combined Distance Vector by Appending the $\mathrm{d}^{\mathrm{PCA}}$ Vector $+\mathrm{d}^{\mathrm{LDA}}$ Vector.

$$
\mathrm{d}=\left\{\mathrm{d}_{1}{ }^{\mathrm{PCA}}, \ldots \ldots, \mathrm{d}_{\mathrm{N}}{ }^{\mathrm{PCA}}, \mathrm{d}_{1}{ }^{\mathrm{LDA}}, \ldots \ldots \mathrm{d}_{\mathrm{N}}{ }^{\mathrm{LDA}}\right\}
$$

3.3.Feature Selection: Feature Selection is employed to determine the optimal features subset by eliminating the least important feature in order to increase the classification accuracy and to reduce the computation cost. In this work, a novel combinational model has been employed for feature selection using Differential Evolution model and Intelligent Water Drops model to generate the less no of optimal features for authentication of user for secure data access of mobile cloud data.

\subsubsection{Differential Evolution Model (DE)}

a. Description of Differential evolution: Differential Evolution is a stochastic population-based optimization algorithm for its efficient search capability of the feature using iterative process. It is mainly a four step processInitialisation, Mutation, Recombination and Selection on the effective computation of the relevant feature for authentication of the user for data access in the mobile cloud. In the initialisation stage a set of population vectors normally equal to three times of data dimension are chosen in a uniformly random fashion (Kingshuk Chakravarty et. al.(2013)). Each of these parameter vectors undergoes mutation, recombination and selection to generate a trial vector. In mutation stage, for a given parameter vector, three parameter vector has been randomly selected that they distinct to each other on face and fingerprint fused vector. In recombination stage each element of the trial vector is generated depending on the value of cross over rate $\varepsilon(0,1)$.

Computing objective Function: In this section, objective function of the Differential Evolution based feature selection has been constructed on basis of assumption of secure data access constraints in the mobile cloud. Initial assumption is that the good feature of the fusion vector should have least variation or distance value between the feature instances of the vector of face and fingerprint which considered as intra vector variation. The objective function for determining the intra vector variation of the finger and face image fusion is as follows.
Objective Function of Feature selection

$$
\left.\mathrm{O}_{\mathrm{i}}\left(\mathrm{x}_{\mathrm{k}}\right)=\sum_{k=0}^{n}\left(\begin{array}{l}
n \\
k
\end{array}\right) x^{k} a^{n-k} \mathrm{v}_{\mathrm{i}}\left(\mathrm{x}_{\mathrm{k}}\right)-\mathrm{v}_{\mathrm{i}} \mathrm{j}\left(\mathrm{x}_{\mathrm{m}+1}\right)\right)
$$

Where $\mathrm{v}_{\mathrm{ij}}\left(\mathrm{x}_{\mathrm{k}}\right)$ denotes the value of $\mathrm{jth}$ feature in the fusion vector and $\mathrm{n}$ is the number of the features in the fusion vector of the multimodal biometric. Each population of the DE algorithm as parameter vector which plays an important role in finding the optimum feature subset for authentication.

\subsection{Feature Classification using Multivariate Linear} Regression: The optimal feature subset obtained on feature selection has been classified using Multivariate Linear Regression classifier for authentication of the user for secure access in the mobile cloud. It is a non parametric method (N. a. Samusdin et. al.(2010)). The method is considered to be the instance based as it predicts the class label for the feature sample by finding $\mathrm{k}$ closest points of the features selected. The $\mathrm{k}$ closest points are predicted by the distance weighting estimation to train feature samples as classes and predict the class label for the sample. Let $\mathrm{X}$ is the optimal feature vector for which the nearest neighbour has to compute which represent the class; each class is Tij may be estimated by distance measure of Euclidean distance. The distance estimation is carried out using Euclidean distance. Distance between the feature samples is Tij which can be represented as.

$$
\mathrm{D}_{\mathrm{i}}(\mathrm{y})=\left\|\mathrm{y}-\mathrm{T}_{\mathrm{ij}}\right\|^{2}
$$

Where $T_{i j}$ is Nearest Neighbour is to optimal feature vector for authentication of user. Determine the category of the features such that category which represents most of the features. In this conclude $\mathrm{T}_{\mathrm{ij}}$ belongs that particular category. In this category is collection of feature or its values to represent individual.

Euclidean distance between two features in fused vector is.

$$
\mathrm{E}_{\mathrm{d}}=\sqrt{(\Delta \mathrm{x})^{2}+(\Delta \mathrm{y})^{2}}
$$

4. Experimental Analysis: In this work, fusion of multimodal biometric for secure access of mobile cloud data using a new combinational model has been experimented. The results for the experiment have been computed and its performance has been evaluated on various setting through $\mathrm{K}$ fold cross validation is described in detail in below sections.

\subsection{Experimental Environment and Dataset Description:}

The experiment was carried out using MATLAB R2018 on window 7 Intel Core i7 CPU with operating frequency of $2.53 \mathrm{G} \mathrm{Hz}$ and a usable memory of $8 \mathrm{~GB}$. The dataset used in this work was acquired from 100 subjects with 20 fingers and face from each subject which produces 500 samples. The images have been taken in three sessions, four images 
each time, at least one week between each session. The image in the database has $1024 * 780$ resolutions and saved as a 12-bit bitmap.

4.2. Training: Images of 25 subjects among 100 subjects are used for training in the 10 fold validation (J. Chai et. al.(2010)). Training of the fingerprint and face recognition model, the fingerprint and face patterns extracted using single dimensional feature vectors through principle Component Analysis and Linear Discriminant Analysis. Table 1 and Table 2 compare the recognition performance of the existing method using PCA LDA Euclidean and PCA_LDA_Cosine as state-of-the-art methods. The stateof-the-art methods are compared by dividing them into non- training-based and training-based methods.

\begin{tabular}{|c|c|c|c|c|c|}
\hline Threshold & 0.1 & 0.2 & 0.01 & 0.03 & 0.05 \\
\hline Accuracy & 0.969543 & 0.971698 & 0.968586 & 0.968586 & 0.968586 \\
\hline FRR & 5.813953 & 2.970297 & 7.5 & 7.5 & 7.5 \\
\hline FAR & 2.702703 & 0 & 0 & 0 & \\
\hline Precision & 98.78049 & 97.0297 & 100 & 100 & 100 \\
\hline Recall & 94.18605 & 97.0297 & 92.5 & 92.5 & 92.5 \\
\hline
\end{tabular}

\begin{tabular}{|c|c|c|c|c|c|}
\hline Threshold & 0.1 & 0.2 & 0.01 & 0.03 & 0.05 \\
\hline Accuracy & 100 & 100 & 100 & 100 & 100 \\
\hline FRR & 0 & 0 & 0 & 0 & 0 \\
\hline FAR & 0 & 0 & 0 & 0 & 0 \\
\hline Precision & 100 & 100 & 100 & 100 & 100 \\
\hline Recall & 100 & 100 & 100 & 100 & 100 \\
\hline
\end{tabular}

In this section, we present experiments to show the effectiveness of the proposed method in combining feature sets extracted from PCA and LDA model on fusion of multimodal biometric images. Since the adjacent pixels in an image are usually correlated (A. M. Martinez et. al.(2001)), the information redundancy can be reduced by downsampling the feature images. In our experiments, the feature images are down sampled PCA and LDA by factor of $\mathrm{n}$. In this $\mathrm{n}$ can be any number between 1 to 5 .
$\mathrm{N}$ - Dimensional feature vector is extracted from each point and the final feature vector is constructed by concatenating the feature vectors of PCA and LDA. However Fused Feature vector (C. Liu et. al. (2016)) improves the recognition which further undergoes feature selection using DE and IWD. Initially parameter setting for the feature selection model using DE and has been tabulated as follows

Table 3. Performance comparison of classifier for multimodal Biometric authentication on optimal feature

\begin{tabular}{|l|c|c|c|c|c|}
\hline Classifiers & Accuracy (\%) & Recall (\%) & Precision (\%) & FRR (\%) & FAR (\%) \\
\hline Euclidean & 96 & 93.6 & 100 & 6.25 & 1.54 \\
\hline KNN & 100 & 100 & 100 & 0 & 0 \\
\hline
\end{tabular}

4.3. Testing phase: For evaluating the performance of the proposed fingerprint authentication on portion of image considered as test image. So, to investigate the differences among fingers appear better than ring fingers in correct classification rate (CCR) but lower than other fingers in FAR(H. Qin et. al.(2011)). Hence, the fingerprint images from different fingers are viewed as from different individuals in the processing.

4.4. Comparative Results of Feature Selection and Feature Recognition: In this section, the following experiments are conducted for acquired image. Furthermore, 
two vectors of the features are concatenated With DCA based feature fusion method. Our proposed algorithm exhibits more outstanding performance than the existing method. In table 3 provides the performance of finger and face authentication technique on various performances metric such as Accuracy, Recall and Precision.

\begin{tabular}{|c|c|c|c|c|c|}
\hline $\begin{array}{l}\text { Number of } \\
\text { user }\end{array}$ & $\begin{array}{l}\text { Number of } \\
\text { training samples }\end{array}$ & Testing & $\begin{array}{c}\text { Classification } \\
\text { accuracy }\end{array}$ & $\begin{array}{c}\text { Training } \\
\text { Time }\end{array}$ & $\begin{array}{c}\text { Testing } \\
\text { Time }\end{array}$ \\
\hline 10 & 30 & 20 & 99 & 1.5 & 0.6 \\
\hline 40 & 120 & 80 & 100 & 5 & 3.3 \\
\hline
\end{tabular}

\begin{tabular}{|c|c|c|c|c|c|}
\hline $\begin{array}{l}\text { Number of } \\
\text { user }\end{array}$ & $\begin{array}{c}\text { Number of } \\
\text { training samples }\end{array}$ & Testing & $\begin{array}{c}\text { Classification } \\
\text { accuracy }\end{array}$ & $\begin{array}{c}\text { Training } \\
\text { Time }\end{array}$ & $\begin{array}{c}\text { Testing } \\
\text { Time }\end{array}$ \\
\hline 10 & 30 & 20 & 100 & 1.2 & 0.8 \\
\hline 40 & 120 & 80 & 100 & 4.8 & 3.2 \\
\hline
\end{tabular}

The MLR classifier is applied to feature selected using DE to recognize the fingerprint and face on the query images. In cosine, the number of neighbors $(\mathrm{K})$ has been used in evaluating the performance accuracy of the mentioned models and is set by one in order to reduce the noise (increase the probability of close neighbor) of the classifier (H. C. Lee et. al.(2010). In addition, DE produced feature set provides the enhanced classification accuracy on employing MLR classifier for finger vein recognition. Performance evaluation for different subject is tabulated in table 5 . Compared to IWD, DE produces the better recognition results with Cosine distance classifier on recognition of user for authentication of secure data access in the mobile cloud environment on every aspect of the recognition performance measures.

\section{CONCLUSION}

We designed and implemented a new combinational model for Multimodal biometric authentication of user to secure data access in mobile cloud environment. In this work, enhanced multimodal authentication system has been obtained on inclusion of feature extraction techniques such as principle component analysis, linear discriminant analysis which produced high relevant features. Moreover those features are concatenated to generate feature fusion through discriminant correlation analysis. Further Differential Evolution has been derived as population based optimization technique to provide optimal subset of features for classification. Finally Multivariate Linear Regression classifier has been applied to categorize the obtained feature and to classify the test or query images. From the experiments, the DE are significantly improved the performance of classification with accuracy of $100 \%$ on user authentication.

\section{REFERENCES}

Chakravarty, K., Das, D., Sinha, A. and Konar, A., 2013, June. Feature selection by differential evolution algorithm-a case study in personnel identification. In 2013 IEEE Congress on Evolutionary Computation (pp. 892899). IEEE.

Chai, J., Liu, H., Chen, B. and Bao, Z., 2010. Large margin nearest local mean classifier. Signal Processing, 90(1), pp.236-248.

Ezhilarasi, G.D., Latchoumi, T.P. and Balamurugan, K., 2020. UIP - A Smart Web Application to Manage Network Environments, Advances in Intelligent systems and computing book series.

Fan, Z., Xu, Y. and Zhang, D., 2011. Local linear discriminant analysis framework using sample neighbors. IEEE Transactions on Neural Networks, 22(7), pp.11191132.

Haghighat, M., Abdel-Mottaleb, M. and Alhalabi, W., 2016. Discriminant correlation analysis: Real-time feature level fusion for multimodal biometric recognition. IEEE Transactions on Information Forensics and Security, 11(9), pp.1984-1996.

Kumar, K.V. and Negi, A., 2008. Novel approaches to principal component analysis of image data based on feature partitioning framework. Pattern Recognition Letters, 29(3), pp.254-264.

Liu, H., Yang, L., Yang, G. and Yin, Y., 2017. Discriminative binary descriptor for finger vein recognition. IEEE Access, 6, pp.5795-5804.

Lee, H.C., Kang, B.J., Lee, E.C. and Park, K.R., 2010. Finger vein recognition using weighted local binary pattern code based on a support vector machine. Journal 
of Zhejiang University SCIENCE C, 11(7), pp.514-524. Liu, F., Yin, Y., Yang, G., Dong, L. and Xi, X., 2014, September. Finger vein recognition with superpixelbased features. In IEEE International Joint Conference on Biometrics (pp. 1-8). IEEE.

Liu, C. and Kim, Y.H., 2016, September. An efficient finger-vein extraction algorithm based on random forest regression with efficient local binary patterns. In 2016 IEEE International Conference on Image Processing (ICIP) (pp. 3141-3145). IEEE.

Martinez, A.M. and Kak, A.C., 2001. Pca versus lda. IEEE transactions on pattern analysis and machine intelligence, 23(2), pp.228-233.

Miura, N., Nagasaka, A. and Miyatake, T., 2007. Extraction of finger-vein patterns using maximum curvature points in image profiles. IEICE TRANSACTIONS on Information and Systems, 90(8), pp.1185-1194.

Mishra, P., Jimmy, L., Ogunmola, G.A., Phu, T.V., Jayanthiladevi, A. and Latchoumi, T.P., 2020, December. Hydroponics cultivation using real time iot measurement system. In Journal of Physics: Conference Series (Vol. 1712, No. 1, p. 012040). IOP Publishing.

Prasath, S. (2020). Probabilistic Mceliece Public-Key Cryptography Based Identity Authentication For Secured Communication In Vanet. Solid State Technology, 63(6), 10167-10182.

Prasath, S. Validating Data Integrity in Steganographed Images using Embedded Checksum Technique. International Journal of Computer Applications, 975, 8887.

Qin, H., Qin, L. and Yu, C., 2011. Region growth-based feature extraction method for finger-vein recognition. Optical Engineering, 50(5), p.057208

Rosdi, B.A., 2016. Finger vein identification based on the fusion of nearest neighbor and sparse representation based classifiers. Indian Journal of Science and Technology, 9(48).

Rosdi, B.A., Shing, C.W. and Suandi, S.A., 2011. Finger vein recognition using local line binary pattern. Sensors, 11(12), pp.11357-11371.

Samsudin, N.A. and Bradley, A.P., 2010. Nearest neighbour group-based classification. Pattern Recognition, 43(10), pp.3458-3467.

Shah-Hosseini, H., 2008. Intelligent water drops algorithm: A new optimization method for solving the multiple knapsack problem. International Journal of Intelligent Computing and Cybernetics.

Venkata Pavan, M., Karnan, B. and Latchoumi, T.P., 2021. PLA-Cu reinforced composite filament: Preparation and flexural property printed at different machining conditions. Advanced Composite Materials, https://doi. org/10.1080/09243046.2021, 1918608.

Wu, J.D. and Liu, C.T., 2011. Finger-vein pattern identification using principal component analysis and the neural network technique. Expert Systems with Applications, 38(5), pp.5423-5427.

Yang, J., Shi, Y. and Jia, G., 2017. Finger-vein image matching based on adaptive curve transformation. Pattern Recognition, 66, pp.34-43.

Yang, G., Xi, X. and Yin, Y., 2012. Finger vein recognition based on (2D) 2 PCA and metric learning. Journal of Biomedicine and Biotechnology. 